\title{
Shoulder Arthroscopy in Conjunction With an Open Latarjet Procedure Can Identify Pathology That May Not Be Accounted for With Magnetic Resonance Imaging
}

\author{
Justin J. Ernat, M.D., M.H.A., Dylan R. Rakowski, B.S., Aaron J. Casp, M.D., \\ Simon Lee, M.D., M.P.H., Annalise M. Peebles, B.A., Jared A. Hanson, B.A., \\ CAPT Matthew T. Provencher, M.D., MC, USNR (Ret), and Peter J. Millett, M.D., M.Sc.
}

\begin{abstract}
Purpose: To review arthroscopic findings at the time of open Latarjet procedures to determine whether preoperative magnetic resonance imaging reports (MRRs) correlate with arthroscopic findings, as well as whether the arthroscopic findings critically affected surgical interventions performed at the time of a Latarjet procedure. Methods: This was a retrospective case series of all patients who received a Latarjet procedure between 2006 and 2018. Patients were excluded if they had inadequate records or underwent revision of a bony reconstruction procedure. Both primary Latarjet procedures and Latarjet procedures for revision of a failed arthroscopic procedure were included. MRRs, arthroscopic findings, and diagnoses were collected, and differences were noted. A "critical difference" was one that affected the surgical intervention in a significantly anatomic or procedural fashion or that affected rehabilitation. Results: In total, 154 of 186 patients $(83 \%)$ were included. Of these, 96 of $154(62 \%)$ underwent revision Latarjet procedures. The average bone loss percentage reported was $20.6 \%$ (range, $0 \%-40 \%$ ). A critical difference between MRR and arthroscopic findings was noted in 60 of 154 patients (39\%), with no difference between Latarjet procedures and revision Latarjet procedures. Of 154 patients, 29 (19\%) received an additional 52 intra-articular procedures for diagnoses not made on magnetic resonance imaging, with no difference between primary and revision procedures. This included biceps and/or SLAP pathology requiring a tenodesis, debridement, or repair; rotator cuff pathology requiring debridement or repair; complex $\left(>180^{\circ}\right)$ labral tears requiring repair; loose bodies; and chondral damage requiring debridement or microfracture. Patients undergoing revision Latarjet procedures were less likely to have bone loss mentioned or quantified in the MRR. Conclusions: Diagnostic imaging may not reliably correlate with diagnostic arthroscopic findings at the time of a Latarjet procedure from both a bony perspective and a soft-tissue perspective. In this series, diagnostic arthroscopy affected the surgical plan in addition to the Latarjet
\end{abstract}

From Steadman Philippon Research Institute, Vail, Colorado, U.S.A. (J.J.E., D.R.R., A.J.C., S.L., A.M.P., J.A.H., P.J.M.); and The Steadman Clinic, Vail, Colorado, U.S.A. (J.J.E., A.J.C., S.L., M.T.P., P.J.M.).

The authors report the following potential conflicts of interest or sources of funding: J.J.E. receives a yearly salary from Steadman Philippon Research Institute (SPRI), outside the submitted work. SPRI exercises special care to identify any financial interests or relationships related to research conducted here. During the past calendar year, SPRI has received grant funding or inkind donations from Arthrex, Department of Defense Office of Naval Research, DJO, Ossur, Siemens, and Smith $\theta$ Nephew. D.R.R. is employed by SPRI, outside the submitted work. A.J.C. receives a yearly salary from SPRI, outside the submitted work. S.L. receives a yearly salary from SPRI, outside the submitted work. A.M.P. is employed by SPRI, outside the submitted work. J.A.H. is employed by SPRI, outside the submitted work. M.T.P. receives intellectual property royalties from Arthrex; is a consultant for Arthrex, Joint Research Foundation, and SLACK; is a speaker for Arthrex; and receives an honorarium from Arthrosurface, outside the submitted work. In addition, M.T.P. is an editorial or governing board member for Arthroscopy, Knee, Orthopedics, and SLACK and is a board or committee member for Arthroscopy Association of North America, American Academy of Orthopaedic Surgeons, American Orthopaedic Society for Sports Medicine, American
Shoulder and Elbow Surgeons, International Society of Arthroscopy, Knee Surgery and Orthopaedic Sports Medicine, San Diego Shoulder Institute, and Society of Military Orthopaedic Surgeons. P.J.M. is a consultant for Arthrex; receives royalties from Arthrex, Springer Publishing, and MedBridge; receives grants from Arthrex; owns stock options in VuMedi; is part owner of ProofPoint Biologics; and receives support from research activities from SPRI, outside the submitted work. In addition, his institution receives funding from Arthrex, Smith $\theta$ Nephew, Siemens, and Össur, outside the submitted work. Full ICMJE author disclosure forms are available for this article online, as supplementary material.

Received April 30, 2021; accepted September 22, 2021.

Address correspondence to Peter J. Millett, M.D., M.Sc., Center for Outcomes-based Orthopaedic Research, Steadman Philippon Research Institute, 181 W Meadow Dr, Ste 1000,Vail, CO 81657,U.S.A.E-mail:drmillett@ thesteadmanclinic.com

(C) 2021 Published by Elsevier Inc. on behalf of the Arthroscopy Association of North America. This is an open access article under the CC BY-NC-ND license (http://creativecommons.org/licenses/by-nc-nd/4.0/).

2666-061X/21651/\$36.00

https://doi.org/10.1016/j.asmr.2021.09.014 
procedure in $19 \%$ of cases. We recommend performing a diagnostic arthroscopy prior to all Latarjet procedures to identify and/or treat all associated intra-articular shoulder pathologies. Level of Evidence: Level IV, diagnostic case series.

$\mathbf{S}^{\text {s }}$ oft-tissue and bony injuries most commonly associated with anterior shoulder instability include the Bankart lesion, bony Bankart lesion, humeral avulsion of the glenohumeral ligament (HAGL), bony humeral avulsion of the glenohumeral ligament (BHAGL), HillSachs lesion, and anterior labral periosteal sleeve avulsion (ALPSA). ${ }^{1}$ Additionally, bony Bankart lesions are present in up to $20 \%$ of first-time dislocations and $90 \%$ of recurrent dislocations, ${ }^{2}$ and humeral bone defects (Hill-Sachs lesions) are found in up to $67 \%$ of firsttime dislocations and $100 \%$ of recurrent dislocations. ${ }^{3-5}$ The aforementioned bony and soft-tissue injuries associated with anterior instability have been extensively studied. ${ }^{6,7}$ Extensive labral, biceps tendon, rotator cuff tendon, bony, and capsular injuries can all occur in conjunction with primary or recurrent anterior shoulder instability. ${ }^{8-11}$ Magnetic resonance imaging (MRI) and computed tomography (CT) are beneficial for identification of pathology seen with anterior shoulder instability; however, identifying combined injuries may be more challenging. ${ }^{12}$ Ricchetti et al. ${ }^{13}$ discussed advanced imaging findings in 46 shoulders with confirmed arthroscopic findings of a pan-labral tear. They found that MRI or magnetic resonance arthrography preoperatively identified a pan-labral tear in only $21.3 \%$ of all studies.

Open procedures with a deltopectoral approach do not always afford easy access to all aspects of the glenohumeral joint. Because of this, diagnostic arthroscopy is frequently performed prior to an open approach to bony reconstruction of the glenoid. MRI and/or CT findings can be confirmed, and unanticipated or additional pathology can be managed as needed. To our knowledge, only 1 study has previously reported on the additional arthroscopic findings present immediately prior to an open Latarjet (OL) reconstruction for recurrent anterior instability with glenoid bone deficiency: Arrigoni et al. ${ }^{14}$ reviewed 33 consecutive patients who matched these criteria. They discovered that $73 \%$ of cases had associated pathologic lesions that were identified and addressed arthroscopically and that likely could not have been discovered or treated optimally during an open deltopectoral approach. These included primarily SLAP lesions $(64 \%)$ but also posterior labral tears, loose bodies, rotator cuff tears, and cartilage injuries. Yiannakopoulos et al. ${ }^{11}$ reported on secondary intra-articular lesions in patients with acute and chronic shoulder instability independent of glenoid bone loss. They found that SLAP lesions did not differ in frequency between acute and chronic cases and were observed at a rate of $20.47 \%$.
The purpose of this study was to review arthroscopic findings at the time of Latarjet procedures to determine whether preoperative magnetic resonance imaging reports (MRRs) correlate with arthroscopic findings, as well as whether the arthroscopic findings critically affected surgical interventions performed at the time of a Latarjet procedure. We hypothesized that arthroscopic findings would have the potential to differ from preoperative MRI findings and that this difference could affect treatment and/or rehabilitation in a significant percentage of patients.

\section{Methods}

This study was approved by our institutional review board (No. 2020-41) prior to initiation. All patients aged 16 to 60 years who underwent a Latarjet procedure performed by 2 senior surgeons (M.T.P. and P.J.M.) between August 2006 and October 2018 were included. Patients were excluded if they did not have preoperative advanced imaging reports, were outside the specified age range, received an allograft bone block transfer procedure (because these were typically reserved for revisions of prior bone block procedures during the query period), did not have an operative report available, underwent revision of a prior bony reconstruction procedure, or did not undergo diagnostic arthroscopy prior to the open portion of the procedure. All procedures were performed with a standard diagnostic arthroscopy in conjunction with the Latarjet procedure under the same general anesthesia, in a sequence that has previously been described. ${ }^{15}$ Any additional pathology identified on arthroscopy was addressed as deemed appropriate prior to initiation of the open portion of the procedure. Demographic data collected included age, laterality, sex, and primary Latarjet procedure versus Latarjet procedure for revision of a prior failed arthroscopic stabilization. Preoperative clinical examination findings in all patients were recorded with particular attention to special testing for the diagnosis of specific bony or soft-tissue injuries. All patients' preoperative MRI studies were reviewed, and the findings, as reported by a musculoskeletal radiology fellowship-trained radiologist, were noted. These were cross-referenced with the patients' operative reports regarding the diagnostic arthroscopic findings and subsequent procedures performed in addition to the Latarjet procedure. Intraoperative bone loss was confirmed by noting the injury and wear pattern associated with flattening of the glenoid rim. This was then measured as a percentage of the glenoid using a 
Table 1. Demographic Findings and Critical Differences Between Imaging and Arthroscopic Findings

\begin{tabular}{|c|c|c|c|c|}
\hline & $\begin{array}{l}\text { All Patients } \\
(\mathrm{N}=154)\end{array}$ & $\begin{array}{c}\text { Primary } \\
\text { Latarjet Procedure } \\
(\mathrm{n}=58) \\
\end{array}$ & $\begin{array}{c}\text { Revision } \\
\text { Latarjet Procedure } \\
(\mathrm{n}=96) \\
\end{array}$ & $P$ Value \\
\hline Average age, yr & 28 & 30 & 27 & $>.1$ \\
\hline Right/left & $81 / 73$ & $23 / 35$ & $58 / 38$ & $<.03^{*}$ \\
\hline Average bone loss (available for 111 of 156 patients), \% & $20.6(0-40)$ & $20.8(0-40)$ & $20.6(0-35)$ & .2 \\
\hline Difference in radiology vs arthroscopic findings, n (\%) & 59 of $154(38.3)$ & 19 of $58(32.8)$ & 40 of $96(41.6)$ & $>.2$ \\
\hline Biceps tenodesis, n (\%) & 5 of $154(3.2)$ & 4 of $58(6.9)$ & 1 of $96(1)$ & $>.06$ \\
\hline SLAP repair or debridement, n (\%) & 9 of $154(5.8)$ & 6 of $58(10.3)$ & 3 of $96(3.1)$ & $>.08$ \\
\hline$>180^{\circ}$ labral tearing with repair or debridement, n (\%) & 5 of $154(3.2)$ & 3 of $58(5.2)$ & 2 of $96(2.1)$ & $>.3$ \\
\hline Rotator cuff procedure, n (\%) & 4 of $154(2.6)$ & 2 of $58(3.5)$ & 2 of $96(2.1)$ & $>.6$ \\
\hline Repair & 1 of $154(0.6)$ & 0 of $58(0)$ & 1 of $96(1)$ & \\
\hline Debridement & 3 of $154(2)$ & 2 of $58(3.4)$ & 1 of $96(1)$ & \\
\hline
\end{tabular}

*Statistically significant $(P<.05)$.

probe and anticipated glenoid width based on a circle method. ${ }^{16}$

Chart reviews for inclusion and exclusion were carried out by 2 research assistants (D.R.R., A.M.P.) and 3 orthopaedic sports medicine fellows (J.J.E., A.J.C., S.L.). The imaging and operative report findings were then acquired and confirmed by 3 orthopaedic sports medicine fellows. Final confirmation was made by 2 sports medicine fellowship-trained orthopaedic surgeons. Given that the data acquired were reported in the chart and given that no independent reviewer interpretation was required, no validation studies were performed.

Patients were stratified based on whether their MRI findings correlated with the procedural findings, and the various additional procedures performed were recorded. A critical difference was noted between MRI findings and arthroscopic findings when the surgical intervention was affected in a significantly anatomic or procedural fashion or in a way that may affect rehabilitation protocols. These findings were, in essence, "under-read" by the radiologist. For example, if a rotator cuff injury that required repair were identified, this would be a critical difference in anatomy requiring intervention that would change both treatment and the rehabilitation protocol. Conversely, removal of old anchors would not affect the surgical plan or the rehabilitation protocol, so this would not be considered "critical." Specifically, when intra-articular pathology was present that could not have been reliably addressed through an open deltopectoral approach, this was noted. Additionally, we noted when the MRI findings were "over-read," that is, when the MRR noted a pathology that was not present in the arthroscopic findings. These were considered separate entities from the critical differences described earlier.

\section{Statistical Analysis}

The incidences of associated intra-articular lesions from radiology reports, as well as new lesions found on diagnostic arthroscopy, were assessed and reported. Statistical analysis with the Fisher exact test was performed to compare critical differences between primary Latarjet procedures and revision Latarjet procedures for failed arthroscopic stabilization to determine whether there was a difference in unanticipated intra-articular procedures, as well as to compare differences in the qualitative identification of bone loss as it pertains to radiologic and arthroscopic findings. $P<.05$ was considered statistically significant.

\section{Results}

A total of 154 of 186 patients (83\%) undergoing the Latarjet procedure met the criteria for analysis. Of these 154 patients, $116(75 \%)$ were male patients; 81 of 154 shoulders $(53 \%)$ were right shoulders; and the average age was 28 years (range, 16-58 years). The reasons for exclusion of 32 patients were as follows: 21 lacked imaging reports or had incomplete imaging reports, 4 had incomplete operative reports, and 7 underwent revision of a previous bone augmentation procedure. The quantifiable bone loss reported in the operative note was available for 111 of 154 patients and averaged $20.6 \%$ (range, $0 \%-40 \%$ ). Of 154 patients, $96(62 \%$ ) underwent the Latarjet procedure for revision of a prior arthroscopic stabilization procedure. 
A critical difference between imaging reports and arthroscopic findings was noted in $38 \%$ of cases (59 of 154), with no difference between primary and revision procedures $(P>.2)$. Specifically, 29 of 154 patients $(18.8 \%)$ underwent a combined additional 52 intraarticular procedures that addressed diagnoses that were not made on MRI (Table 1), with no difference between primary and revision procedures $(P>.6)$. This does not include removal of previously placed anchors, which was performed in 49 of 154 patients $(32 \%)$. Additional planned procedures not mentioned in Table 1 included remplissage in 2 of 154 patients $(1.3 \%$, both primary operations), humeral head bone grafting procedures in 2 of $154(1.3 \%$, both primary operations), and axillary nerve decompression in 2 of 154 ( $1.3 \%$, both revision operations). A breakdown of all imaging diagnoses and arthroscopic diagnoses is provided in Table 2. Imaging reports did not mention or quantify bone loss in $22 \%$ of cases ( 34 of 154). This was more likely to occur in a revision setting $(P<.05)$.

There was an over-read in the MRR as compared with the arthroscopic findings in 30 of 154 patients (19.5\%). This included MRR mentions of SLAP tears in 10 of 154 patients $(6.5 \%)$, rotator cuff tears in 8 of $154(5.2 \%)$, biceps tendon pathology in 7 of $154(4.5 \%)$, rotator cuff and SLAP tears in 2 of $154(1.3 \%)$, labral tearing greater than $180^{\circ}$ in 2 of $154(1.3 \%)$, and an additional posterior labral tear in 1 of $154(0.6 \%)$-none of which were diagnosed arthroscopically.

\section{Discussion}

The most important finding of this study was that almost $40 \%$ of patients receiving a Latarjet procedure, whether as a primary surgical procedure or as a revision of a previous arthroscopic stabilization, had arthroscopic findings that were different from MRI findings. This included $18.6 \%$ of patients in whom the difference in findings resulted in a critical change in intra-articular or arthroscopic treatment from either an anatomic, pathologic, or rehabilitative perspective. Thus, our hypothesis was confirmed. There was no statistically significant difference in this occurrence between the primary and revision settings. Additionally, in $22.7 \%$ of cases, the MRI report did not mention or quantify bone loss, and this was almost twice as common when the Latarjet procedure was performed for revision of a prior failed arthroscopic stabilization procedure.

The value of arthroscopy prior to an open modified Latarjet procedure has been previously studied. Arrigoni et al. ${ }^{14}$ reported diagnostic arthroscopic findings in 33 consecutive cases undergoing either primary Latarjet procedures $(45 \%)$ and Latarjet procedures for revision of prior stabilization procedures $(55 \%)$ in patients with an average age of 25 years. All patients had a minimum of $25 \%$ glenoid bone loss. The authors noted that $73 \%$ of patients had associated intra-articular pathologies requiring treatment, the most common of which were SLAP tears (type II SLAP tears in 64\%). These were all identified and repaired arthroscopically with suture anchors. Additional injuries included a posterior labral tear in 1 patient; grade 4 chondromalacia in 2; large loose bodies requiring removal in 2 ; and rotator cuff tears in 2, with debridement in 1 and repair in 1 . In our study, only approximately $6 \%$ of patients required treatment of a SLAP lesion including both debridement and repair. An additional approximately $3 \%$ of patients underwent a biceps tenodesis. We identified far less biceps or superior labral pathology than Arrigoni et al. even after combining all of these patients' pathologies. In a study by Yiannakopoulos et al., ${ }^{11}$ SLAP lesions (types I-IV) were identified in $20 \%$ of 127 cases of anterior shoulder instability, with no significant difference between acute and chronic cases, which is more reflective of our findings. Regarding other unanticipated injuries requiring treatment, our results are more correlative with those of Arrigoni et al., given that 7\% of our cases had chondral injuries requiring treatment versus $6.1 \%$ in their series; $9 \%$ versus $6.1 \%$ underwent unanticipated loose body removal; and $7.1 \%$ versus $6.1 \%$ received unanticipated treatment for a rotator cuff tear. Yiannakopoulos et al. found a partialthickness articular rotator cuff tear in 14 patients: 12 with chronic dislocations and 2 with acute dislocations $(11.53 \%$ vs $8.69 \%)$. None of these tears required a repair, and all were debrided.

We sought to compare the arthroscopic findings with the preoperative radiology reports. A difference between the radiology report and the arthroscopic findings was noted in $38 \%$ of patients, with no difference between primary and revision Latarjet procedures, although this only led to a critical change in surgical treatment in $18.6 \%$ of patients, as noted earlier. However, we believe that this difference in radiologic versus arthroscopic findings is noteworthy for several reasons: First, when using MRI as a diagnostic tool in the setting of shoulder instability, it is important to note, when counseling the patient, that there may be additional injuries either unaccounted for or over-read by the radiologist and that any of these discrepancies can be addressed appropriately at the time of diagnostic arthroscopy. This has been documented before in cases of recurrent or complex shoulder instability. For example, in diagnosing complex labral lesions, Ricchetti et al. ${ }^{13}$ found that MRI or magnetic resonance arthrography was only able to identify pan-labral lesions in $21.3 \%$ of those cases confirmed at the time of arthroscopy. Second, when preoperatively planning for an $\mathrm{OL}$, the surgeon should be prepared to encounter unanticipated pathology at the time of diagnostic arthroscopy. This could entail additional anchors, suture-passing devices, patient positioning, and so on in 
Table 2. Summary of Imaging and Arthroscopic Findings at Time of Latarjet Procedure

\begin{tabular}{|c|c|c|c|c|c|c|c|}
\hline & \multicolumn{2}{|c|}{ All Patients $(\mathrm{N}=156)$} & \multicolumn{2}{|c|}{ Primary Latarjet Procedure $(\mathrm{n}=59)$} & \multicolumn{2}{|c|}{ Revision Latarjet Procedure $(\mathrm{n}=97)$} & \multirow[b]{2}{*}{$P$ Value ${ }^{*}$} \\
\hline & MRI Diagnosis & Arthroscopic Diagnosis & MRI Diagnosis & Arthroscopic Diagnosis & MRI Diagnosis & Arthroscopic Diagnosis & \\
\hline Biceps pathology & 15 of $154(9.7)$ & 7 of $154(4.5)$ & 9 of $58(15.5)$ & 4 of $58(6.9)$ & 6 of $96(6.3)$ & 3 of $96(3.1)$ & $\begin{array}{l}.9 \\
.15\end{array}$ \\
\hline Bankart tear & 108 of $154(70.1)$ & 99 of $154(64.3)$ & 40 of $58(69.0)$ & 37 of $58(63.8)$ & 68 of $96(70.8)$ & 62 of $96(64.69)$ & $\begin{array}{l}.8 \\
.8\end{array}$ \\
\hline$>180^{\circ}$ tear & 6 of $154(3.9)$ & 5 of $154(3.2)$ & 3 of $58(5.2)$ & 3 of $58(5.2)$ & 3 of $96(3.1)$ & 2 of $96(2.1)$ & $\begin{array}{l}.6 \\
.3\end{array}$ \\
\hline Bony Bankart lesion & 34 of $154(22.1)$ & 15 of $154(9.7)$ & 24 of $58(41.4)$ & 10 of $58(17.2)$ & 10 of $96(10.4)$ & 5 of $96(5.2)$ & $\begin{array}{l}.001^{\dagger} \\
.02^{\dagger}\end{array}$ \\
\hline Hill-Sachs lesion & 81 of $154(52.6)$ & 63 of $154(41)$ & 37 of $58(63.8)$ & 29 of $58(50.0)$ & 45 of $96(46.9)$ & 34 of $96(35.4)$ & $\begin{array}{l}.06 \\
.06\end{array}$ \\
\hline Rotator cuff tear & 11 of $154(7.1)$ & 4 of $154(2.6)$ & 9 of $58(15.5)$ & 2 of $58(3.4)$ & 2 of $96(2.1)$ & 2 of $96(2.1)$ & $\begin{array}{l}.003^{\dagger} \\
.6\end{array}$ \\
\hline SLAP tear & 14 of $154(9.1)$ & 10 of $154(6.5)$ & 7 of $58(12.1)$ & 6 of $58(10.3)$ & 7 of $96(7.3)$ & 4 of $96(4.2)$ & $\begin{array}{l}.3 \\
.2\end{array}$ \\
\hline Loose bodies & 16 of $154(10.4)$ & 22 of $154(14.3)$ & 7 of $58(12.1)$ & 7 of $58(12.1)$ & 9 of $96(9.4)$ & 15 of $96(15.6)$ & $\begin{array}{l}.5 \\
.6\end{array}$ \\
\hline Subscapularis tear & 1 of $154(0.7)$ & 4 of $154(2.6)$ & 0 of $58(0)$ & 1 of $58(1.7)$ & 1 of $96(1)$ & 3 of $96(3.1)$ & $\begin{array}{l}>.999 \\
>.999\end{array}$ \\
\hline Chondral damage & 21 of $154(13.6)$ & 15 of $154(9.7)$ & 11 of $58(19.0)$ & 5 of $58(8.6)$ & 11 of $96(11.5)$ & 10 of $96(10.4)$ & $\begin{array}{l}.2 \\
.7\end{array}$ \\
\hline HAGL & 2 of $154(1.3)$ & 0 of $154(0)$ & 0 of $58(0)$ & 0 of $58(0)$ & 2 of $96(2.1)$ & 0 of $96(0)$ & $\begin{array}{c}.5 \\
>.999\end{array}$ \\
\hline
\end{tabular}

NOTE. Data are presented as number (percentage).

HAGL, humeral avulsion of glenohumeral ligament; MRI, magnetic resonance imaging.

*The upper $P$ value compares MRI diagnoses between primary Latarjet and revision Latarjet procedures; the lower $P$ value compares arthroscopic diagnoses between primary Latarjet and revision Latarjet procedures.

${ }^{\dagger}$ Statistically significant $(P<.05)$. 
the event that an unanticipated labral tear, biceps pathology, or rotator cuff injury is found.

Another key finding as it pertains to the comparison of MRI reports and arthroscopic findings is the discrepancy in reported bone loss-or lack thereof-in the preoperative imaging reports. The imaging findings failed to mention bone loss in $22 \%$ of all patients, and this was more common in revision OL cases, in which this was evident in $27.8 \%$ of patients. Although some surgeons may rely on CT or 3-dimensional CT to define bone loss in the setting of anterior shoulder instability, MRI has been shown to be equally reliable when formatted correctly, with a maximal difference of $1.3 \%$ compared with CT. ${ }^{17}$ However, if the images are not appropriately formatted or if the reader is not specifically attempting to identify bone loss, then it could easily be unaccounted for or under-quantified. Thus, it is imperative for surgeons to be cognizant of the presence of bone loss and to request appropriately formatted images or order a CT scan or 3-dimensional CT scan as they see fit for their practice because this could potentially lead to surgical planning implications for the Latarjet procedure, such as classic versus congruent-arc techniques, or even the necessity of an allograft to plan for the appropriate amount of bony restoration. ${ }^{18,19}$

\section{Limitations}

This study is not without limitations. First, we used the MRR for our comparison to arthroscopic findings. This was a standardized method that could be reliably reproduced from each patient's record; however, it did not account for the surgeon's interpretation of the imaging, which can often differ from the radiologist report, or the surgeon may not be routinely reading the report. ${ }^{20,21}$ Thus, there is the possibility that the critical differences observed, as well as the critical changes in treatments performed, may be different depending on surgeon interpretation and/or experience. Second, in this patient cohort, we did not account for the number of instability episodes as it pertains to the MRI or arthroscopic findings. It is well known that recurrent instability can be a risk factor for additional humeral, glenoid, or soft-tissue injuries, and this could be an influential factor in predicting which patients are at risk of concomitant injuries at the time of the Latarjet procedure. ${ }^{22,23}$ In general, however, most patients indicated for primary Latarjet procedures-and certainly for revision Latarjet procedures-present with a history of recurrent instability. Additionally, this was not the primary aim of our study; the aim was to outline the differences between radiology and arthroscopic findings and thus assist with surgeon counseling and preparation. Finally, although all films were reviewed by a musculoskeletal radiologist, multiple radiologists were involved over the study course of more than 12 years. Although this could create some interpretation bias in the MRR, we suggest that this is an adequate reflection of the reporting that one may experience in the general population because it is rare to have a single radiologist read all films for a practice. Thus, we believe that this had limited impact on the conclusions we present.

\section{Conclusions}

Diagnostic imaging may not reliably correlate with diagnostic arthroscopic findings at the time of a Latarjet procedure from both a bony perspective and a softtissue perspective. In this series, diagnostic arthroscopy affected the surgical plan in addition to the Latarjet procedure in 19\% of cases. We recommend performing a diagnostic arthroscopy prior to all Latarjet procedures to identify and/or treat all associated intraarticular shoulder pathologies.

\section{References}

1. Galvin JW, Ernat JJ, Waterman BR, Stadecker MJ, Parada SA. The epidemiology and natural history of anterior shoulder instability. Curr Rev Musculoskelet Med 2017;10:411-424.

2. Taylor DC, Arciero RA. Pathologic changes associated with shoulder dislocations. Arthroscopic and physical examination findings in first-time, traumatic anterior dislocations. Am J Sports Med 1997;25:306-311.

3. Owens BD, Nelson BJ, Duffey ML, et al. Pathoanatomy of first-time, traumatic, anterior glenohumeral subluxation events. J Bone Joint Surg Am 2010;92:1605-1611.

4. Griffith JF, Antonio GE, Yung PSH, et al. Prevalence, pattern, and spectrum of glenoid bone loss in anterior shoulder dislocation: CT analysis of 218 patients. AJR Am J Roentgenol 2008;190:1247-1254.

5. Widjaja AB, Tran A, Bailey M, Proper S. Correlation between Bankart and Hill-Sachs lesions in anterior shoulder dislocation. ANZ J Surg 2006;76:436-438.

6. Gartsman GM, Roddey TS, Hammerman SM. Arthroscopic treatment of anterior-inferior glenohumeral instability. Two to five-year follow-up. J Bone Joint Surg Am 2000;82-A:991-1003.

7. Hintermann B, Gächter A. Arthroscopic findings after shoulder dislocation. Am J Sports Med 1995;23:545-551.

8. Snyder SJ, Karzel RP, Del Pizzo W, Ferkel RD, Friedman MJ. SLAP lesions of the shoulder. Arthroscopy 1990;6:274-279.

9. Ernat JJ, Yheulon CG, Shaha JS. Arthroscopic repair of 270- and 360-degree glenoid labrum tears: A systematic review. Arthroscopy 2020;36:307-317.

10. Atef A, El-Tantawy A, Gad H, Hefeda M. Prevalence of associated injuries after anterior shoulder dislocation: A prospective study. Int Orthop 2016;40:519-524.

11. Yiannakopoulos CK, Mataragas E, Antonogiannakis E. A comparison of the spectrum of intra-articular lesions in acute and chronic anterior shoulder instability. Arthroscopy 2007;23:985-990. 
12. Kompel AJ, Li X, Guermazi A, Murakami AM. Radiographic evaluation of patients with anterior shoulder instability. Curr Rev Musculoskelet Med 2017;10:425-433.

13. Ricchetti ET, Ciccotti MC, Ciccotti MG, Williams GR, Lazarus MD. Sensitivity of preoperative magnetic resonance imaging and magnetic resonance arthrography in detection of panlabral tears of the glenohumeral joint. Arthroscopy 2013;29:274-279.

14. Arrigoni P, Huberty D, Brady PC, Weber IC, Burkhart SS. The value of arthroscopy before an open modified Latarjet reconstruction. Arthroscopy 2008;24:514-519.

15. Katthagen JC, Anavian J, Tahal DS, Millett PJ. Arthroscopic remplissage and open Latarjet procedure for the treatment of anterior glenohumeral instability with severe bipolar bone loss. Arthrosc Tech 2016;5:e1135-el141.

16. Hamamoto JT, Leroux T, Chahla J, et al. Assessment and evaluation of glenoid bone loss. Arthrosc Tech 2016;5: e947-e951.

17. Gyftopoulos S, Hasan S, Bencardino J, et al. Diagnostic accuracy of MRI in the measurement of glenoid bone loss. AJR Am J Roentgenol 2012;199:873-878.

18. Gilat R, Haunschild ED, Lavoie-Gagne OZ, et al. Outcomes of the Latarjet procedure versus free bone block procedures for anterior shoulder instability: A systematic review and meta-analysis. Am J Sports Med 2021;49: 805-816.

19. Boons HW, Giles JW, Elkinson I, Johnson JA, Athwal GS, Classic versus congruent coracoid positioning during the Latarjet procedure: An in vitro biomechanical comparison. Arthroscopy 2013;29:309-316.

20. Kruger P, Lynskey S, Sutherland A. Are orthopaedic surgeons reading radiology reports? A Trans-Tasman Survey. J Med Imaging Radiat Oncol 2019;63:324-328.

21. van Grinsven S, Nijenhuis TA, Konings PC, van Kampen A, van Loon CJM. Are radiologists superior to orthopaedic surgeons in diagnosing instabilityrelated shoulder lesions on magnetic resonance arthrography? A multicenter reproducibility and accuracy study. J Shoulder Elbow Surg 2015;24: 1405-1412.

22. Krych AJ, Sousa PL, King AH, Morgan JA, May JH, Dahm DL. The effect of cartilage injury after arthroscopic stabilization for shoulder instability. Orthopedics 2015;38: e965-e969.

23. Denard PJ, Dai X, Burkhart SS. Increasing preoperative dislocations and total time of dislocation affect surgical management of anterior shoulder instability. Int J Shoulder Surg 2015;9:1-5. 